\section{Conservative Management of Sclerosing Mucoepidermoid Carcinoma with Eosinophilia of the Thyroid Gland: 12 Years Follow-Up after an Atypical Initial Therapy}

Keywords: Sclerosing mucoepidermoid carcinoma; Thyroid; Incomplete thyroidectomy

\begin{abstract}
Objective: Sclerosing Mucoepidermoid Carcinomas (SMEC) are low-grade malignant tumors with both mucinous and squamous differentiation representing less than $1 \%$ of thyroid malignancies. SMEC are sometimes associated with Hashimoto's thyroiditis and eosinophilia and are more common in females. In rare cases, they have showed a more aggressive behavior with local invasion of the surrounding tissues, trachea, esophagus, or lymph nodes. Distant metastasis to lungs, liver mediastinum, and bone have also been described. SMEC are usually not responsive to Radioactive lodine Ablation (RAIA). Despite the generally low risk of recurrence or death, total thyroidectomy and neck dissection is the recommended therapy.

Methods: We report the case of a 41 year old female with a SMEC of the thyroid. Hemithyroidectomy without lymphadenectomy was performed. Pathology showed a nodule with a maximum diameter of 1.5 centimeters contacting the capsule. Tumor cells expressed TTF-1, CEA and CK7. No expression for thyroglobulin, calcitonin, cromogranin or CK20 was observed.

Results: Complete resection of the thyroid was not performed in this case, and the patient was not treated with radioactive iodine postoperatively. After 12 years of follow-up, the patient did not develop residual disease, neither recurrent loco regional nor metastatic disease. Conclusion: Sclerosing mucoepidermoid carcinoma was treated with hemithyroidectomy, no lymphadenectomy was performed. Despite the size of the nodule and the capsular invasion, the patient had a favourable outcome after a twelve year follow-up. This kind of approach should be taken into account in selected patients.
\end{abstract}

\section{Introduction}

Thyroid nodules are a very common clinical problem in Endocrinology. The identification of thyroid cancer is nowadays a priority as its prevalence is increasing.

A Sclerosing Mucoepidermoid Carcinoma (SMEC) is a rare form of thyroid carcinoma. There are only 40 cases described in the literature and it has recently been recognized by the World Health Organization (WHO) as a primary malignant neoplasm of the thyroid gland [1]. It is a tumor that appears more frequently in adult women, between 35 and 71 years of age [2]. The most common presenting sign is a painless neck mass or a nodule on thyroid image, usually cold on scintigraphy. Although its growth is usually slow, some rare cases of rapidly growing tumors have been described [3]. Significant fibrosis and stroma full of eosinophilic leukocytes are observed histologically

\section{Advances in}

Diabetes \& Endocrinology

\author{
Maria Requena*, Juan C. Percovich, Marcel E. \\ Sambo and Victor M. Andía \\ Department of Endocrinology and Nutrition, Gregorio Marañón \\ General University Hospital, Spain

\section{*Address for Correspondence} \\ Maria Requena, Department of Endocrinology and Nutrition, Gregorio Marañón \\ General University Hospital, C/Doctor Esquerdo, 46. 28007 Madrid, Spain, Tel: \\ + 34 915868112; E-mail: maria.reqang@gmail.com \\ Submission: May 25, 2017 \\ Accepted: July 11, 2017 \\ Published: July 18, 2017 \\ Copyright: () 2017 Requena M, et al. This is an open access article \\ distributed under the Creative Commons Attribution License, which \\ permits unrestricted use, distribution, and reproduction in any medium, \\ provided the original work is properly cited.
}

[2]. The initial fine-needle aspiration is usually inconclusive [4]. The histogenesis of SMEC is unclear and remains under debate [5]. Sclerosing mucoepidermoid carcinoma is often associated with Hashimoto's thyroiditis $[4,6]$, although it has been described that this type of thyroid tumor is not always associated with thyroiditis [2].

The usual behavior of SMEC, as described in the literature, is indolent, and patients frequently live for long periods after the diagnosis, with a range of 1 to 15 years [4]. However, some cases have been described with a more aggressive biological behavior, with local invasion to surrounding tissues, esophagus, trachea or lymph nodes. Distant metastasis to lungs, mediastinum, liver and bone has rarely been identified. Papillary carcinoma frequently shows areas of squamous change, however, it can be distinguished from SMEC by its positivity for thyroglobulin. Medullary carcinoma can also present some squamous and mucinous areas, but it is positive to calcitonin staining which can differentiate it from SMEC [2].

The treatment described in most of the studies is a complete thyroidectomy and lymph node dissection, with or without radiotherapy, chemotherapy, or iodine ablation.

\section{Case Report}

A 41-year-old woman with no remarkable medical history was sent to our endocrinology clinic to evaluate the presence of a thyroid nodule. Physical examination showed a palpable mass in the right lobe of the thyroid. An ultrasound scan was performed, which revealed a tumor with a maximum diameter of $1.5 \mathrm{~cm}$. Thyroid function was normal.

Fine-needle aspiration cytology was performed, which showed the presence of follicular and papillary proliferating cells in a lymph node. A malignant tumor was suspected.

A right hemithyroidectomy was performed on July 2004. The resected thyroid tissue measured $4 \times 2 \times 2 \mathrm{~cm}$ and weighed 6.8 grams, with a tumor measuring $1.5 \times 1 \times 1 \mathrm{~cm}$, and no capsule around it. Parathyroid glands were preserved. In the operating room, surgeons 
Citation: Requena M, Percovich JC, Sambo ME, Andía VM. Conservative Management of Sclerosing Mucoepidermoid Carcinoma with Eosinophilia of the Thyroid Gland: 12 Years Follow-Up after an Atypical Initial Therapy. Adv Diabetes Endocrinol 2017;2(1): 2.

suggested that macroscopically it seemed to be a benign lesion. There were no complications after surgery.

In this case, the tumor contacted the thyroid capsule but did not extend further; it was therefore staged as T1.

The histopathology report showed a sclerosing mucoepidermoid carcinoma in contact with the thyroid capsule. Microscopically, the tumor was composed of numerous nests of squamous cells, glandular structures and papillary formations of solid cells in a sclerotic stroma with numerous eosinophils. Tumor cells presented an irregular nucleus, open nuclear chromatin and moderate eosinophilic cytoplasm in a sclerotic background of chronic inflammatory tissue. The surrounding thyroid tissue showed lymphocytic thyroiditis. The dissected cervical lymph nodes were negative for malignancy. The use of molecular markers revealed positivity to TTF, CEA, and CK7, whereas no expression for thyroglobulin, calcitonin, CK20, chromogranin, or Alcian Blue was observed. The Ki-67 labeling index was not analyzed.

In this case, thyroidectomy was not completed and the patient was not treated postoperatively with radioactive iodine.

During medical reviews in the following years, ultrasound did not reveal tumor recurrence or any suspicious nodes. The patient received suppressive levothyroxine therapy for 8 years and is presently taking $50 \mathrm{mcg}$ of levothyroxine daily to control the underlying hypothyroidism.

\section{Discussion}

Total thyroidectomy with central neck dissection is the elective therapy for this type of tumor [7]. It is important to remark that it does not usually respond to Radioactive Iodine Ablation (RAIA), and although other treatment modalities such as external beam radiotherapy, carboplatin and doxorubicin have been used, none of them have been very successful [8].

Recent studies suggest that, as this tumor is usually associated with Hashimoto's thyroiditis, it probably originates from the metaplastic squamous nests typical of Hashimoto's thyroiditis histology, although other authors suggest that its origin is in the follicular cells [1].

In addition, some cases of aggressive tumors have been published. Chung et al. reported a case of a 57-year-old woman from Korea, who, two years after total thyroidectomy, underwent radical neck dissection because of a recurrent mass. After radiation therapy, a laryngectomy and esophagectomy was performed due to a recurrent carcinoma in the esophageal wall [9]. These authors also reported an additional case of SMEC with metastasis in regional lymph nodes and esophagus [8]. Another case, reported by Purohit et al. described a 70 -year-old woman with kidney metastasis from a SMEC treated with total thyroidectomy, total laryngectomy, and bilateral neck dissection. She also received 150 mci of Radioactive Iodine Ablation [8]. Also, Franssila et al. describe a case of SMEC in which the primary tumor was confined within the thyroid capsule and presenting anaplastic areas invading surrounding structures. This patient died 13 months after diagnosis [10]. Polonia et al. describe a 13-year-old girl with a 2.5 $\mathrm{cm}$ nodule in the left lobe of the thyroid that histologically resulted in SMEC. A cervical lymph node metastasis was identified. The patient was treated with radioactive iodine [11].

BRAF mutation is the most common genetic alteration in thyroid cancer, occurring in $45 \%$ of sporadic papillary thyroid cancers. It is associated with poor clinic pathological outcomes and is an independent molecular prognostic marker in risk evaluation of thyroid cancer [8]. It would be interesting to know its usefulness for this specific tumor; however, it has not been studied yet.

This rare type of thyroid tumor is unique, with a different pathologic classification and also biologically different from the others. A standardized treatment is needed although a consensus or agreement has not yet been made. Additionally, the treatment of metastatic disease is not defined. It is important to find specific clinical, radiological or histological indicators, or molecular markers that will help us predict prognosis and monitor the illness.

\section{Conclusion}

Sclerosing Mucoepidermoid Carcinoma is an infrequent tumor of the thyroid. It shows a female predominance and a relatively benign clinical course, most of the reported cases are low-grade tumors [5], but it can also be very aggressive. Histological studies with molecular markers are needed. Total thyroidectomy and neck dissection is the therapy of choice, based on the irregular outcome of some cases. Despite the initial characteristics of the nodule, our patient had a favorable outcome throughout the 12 years of follow-up after a hemithyroidectomy without lymphadenectomy.

\section{References}

1. Pantola C, Kala S, Athar M, Thakur S (2016) Sclerosing mucoepidermoid carcinoma with eosinophilia of the thyroid: a cytological dilemma. J Cytol 33: 37-39.

2. Baloch ZW, Solomon AC, LiVolsi VA (2000) Primary mucoepidermoid carcinoma and sclerosing mucoepidermoid carcinoma with eosinophilia of the thyroid gland: a report of nine cases. Mod Pathol 13: 802-807.

3. Chan JK, Albores-Saavedra J, Battifora H, Carcangiu ML, Rosai J (1991) Sclerosing mucoepidermoid thyroid carcinoma with eosinophilia. A distinctive low-grade malignancy arising from the metaplastic follicles of hashimotos thyroiditis. Am J Surg Pathol 15: 438-448.

4. Shehadeh NJ, Vernick J, Lonardo F, Madan SK, Jacobs JR, et al. (2004) Sclerosing mucoepidermoid carcinoma with eosinophilia of the thyroid: case report and review of the literature. Am J Otolaryngol 25: 48-53.

5. Lopez JI, Gaafar A, Garmendia M, Velasco V, Ortega FJ, et al. (2008) Sclerosing mucoepidermoid carcinoma of the thyroid gland: cytohistological findings of a case. Hematol Oncol Stem Cell Ther 1: 62-65.

6. Yang SI, Park KK, Jung MJ, Kim JH (2014) Primary sclerosing mucoepidermoid carcinoma of the thyroid: a case report and review of the literature. Head Neck Oncol 6: 43.

7. American Thyroid Association (ATA) Guidelines Taskforce on Thyroid Nodules and Differentiated Thyroid Cancer, Cooper DS, Doherty GM, Haugen BR, Kloos RT, et al. (2009) Revised American Thyroid Association management guidelines for patients with thyroid nodules and differentiated thyroid cancer. Thyroid 19: 1167-1214.

8. Purohit D, Welch BJ, Sukumar S (2011) A case of sclerosing mucoepidermoid carcinoma (SMECE) of the thyroid gland with a BRAF mutation. The Endocrine Society's 93rd Annual Meeting \& Expo, Boston, USA, pp-634.

9. Chung J, Lee SK, Gong G, Kang DY, Park JH, et al. (1999) Sclerosing mucoepidermoid carcinoma with eosinophilia of the thyroid glands: a case report with clinical manifestation of recurrent neck mass. J Korean Med Sci 14: 338-341.

10. Franssila KO, Harach HR, Wasenius VM (1984) Mucoepidermoid carcinoma of the thyroid. Histopathology 8: 847-860.

11. Poland A, Santos L, Eloy C (2012) Sclerosing mucoepidermoid carcinoma of the thyroid in a 13 year-old female. Virchows Archiv 461: S104. 